\title{
Office hysteroscopy after ultrasonographic diagnosis of thickened endometrium in postmenopausal patients
}

\author{
Alexandra Cordeiro $\cdot$ Raquel Condeço • Carla Leitão • \\ Filomena Sousa - Susana Coutinho $\cdot$ Maria do Carmo Silva • \\ Maria José Bernardo $\cdot$ Ricardo Mira
}

Received: 26 December 2008 / Accepted: 24 March 2009/Published online: 16 April 2009

(C) Springer-Verlag 2009

\begin{abstract}
The aim of our study was to access office hysteroscopy results in postmenopausal patients with thickened endometrium. A retrospective descriptive study was carried out on 245 postmenopausal patients submitted to office hysteroscopy after sonographic diagnosis of thickened endometrium in 20 consecutive months. Women were evaluated for age, hormonal therapy, hysteroscopic findings, procedure duration, complications and associated pain, and histological diagnosis. Patients with and without uterine bleeding were considered separately. Symptomatic patients were older and had longer procedure duration. The most frequent hysteroscopic finding was endometrial polyp in both groups. Pain was subjectively assessed in a numeric scale from 0 to 10 and median value was 4 . There were no complications reported. Global neoplasia rate was $2.9 \%$ for asymptomatic patients and $16.4 \%$ for symptomatic ones $(p<0.05)$. Thickened endometrium with postmenopausal metrorrhagia gave patients a significantly higher risk for neoplasia and hyperplasia.
\end{abstract}

Keywords Office hysteroscopy · Endometrial neoplasms . Endometrial hyperplasia $\cdot$ Menopause

\section{Introduction}

Endometrial cancer is known to be the most common malignancy of the female genital tract in the developed world. It occurs primarily after menopause and in most cases presents with vaginal bleeding [1]. However, post-

A. Cordeiro $(\bowtie) \cdot$ R. Condeço $\cdot$ C. Leitão $\cdot$ F. Sousa $\cdot$

S. Coutinho $\cdot$ M. d. C. Silva $\cdot$ M. J. Bernardo $\cdot$ R. Mira

Department of Gynecology and Obstetrics, Dona Estefânia

Hospital, Centro Hospitalar Lisboa Central,

Lisbon, Portugal

e-mail: alexandra.cordeiro@sapo.pt menopausal vaginal bleeding is associated with endometrial neoplasia in only about $10 \%$ to $30 \%$ of the cases [1-3].

Transvaginal ultrasound is the imaging procedure of choice to evaluate patients with postmenopausal bleeding. The most important sonographic sign of endometrial carcinoma is thickened endometrium. In fact, with an endometrial thickness of $5 \mathrm{~mm}$ or less, the chance of a patient having endometrial cancer is about $1 \%[2,4]$. So transvaginal pelvic ultrasound can be used as a screening tool for detection of endometrial pathology in postmenopausal women.

The finding of an endometrial thickness greater than $4 \mathrm{~mm}$, a polypoid endometrial mass, or a collection of fluid within the uterus requires further evaluation, namely endometrial biopsy. Hysteroscopy for thickened endometrium investigation after menopause is now widespread $[5,6]$. The new generation of hysteroscopes combines miniaturization with excellent image resolution and surgical ability. The use of five French bipolar electrodes working in normal saline solution, like the Versapoint ${ }^{\circledR}$ system (Gynecare ${ }^{\circledR}$ Inc., USA), allows most of the pathology to be safely diagnosed and operated at one outpatient session $[7,8]$. At present time, hysteroscopy can be done in an office setting, providing information that cannot be obtained by blind sampling and with high accuracy for uterine cavity pathology diagnosis [5].

The aim of this study was to access office hysteroscopy results in postmenopausal patients with sonographic thickened endometrium and comparing the diagnostic outcomes regarding presence or absence of abnormal bleeding.

\section{Materials and methods}

A retrospective analysis of all office hysteroscopies performed in our institution for a period between January 
2005 and August 2006 was carried out (total $=814$ ). The setting for these procedures was a gynecologic ambulatory unit in a central hospital.

Women $(n=245)$ included in the present study met the following inclusion criteria: (1) menopausal for at least 1 year; (2) previous pelvic transvaginal ultrasound with evidence of increased endometrial thickness; (3) primarily proposed for office hysteroscopy to ascertain endometrial cavity.

Two separate groups were then considered: group A consisted of 172 patients with asymptomatic thickened endometrium; and group B consisted of 73 patients with increased endometrial thickness presenting with uterine bleeding (symptomatic).

The ultrasonographic evaluations were performed at several different centers by different operators; abdominal and transvaginal 2D probes were always combined. Greatest endometrium thickness was measured on a midline sagittal uterus scan, including both anterior and posterior endometrial layers and excluding the surrounding hypoechoic halo. The considered cut-off point was an endometrial thickness superior to $4 \mathrm{~mm}$.

Patients were evaluated for several parameters: age, hormonal therapy, pre-procedure preparation and anesthesia, hysteroscopic findings and procedures, procedure duration, associated pain and complications, and histopathological diagnosis.

Diagnostic and operative hysteroscopies were performed with a $5.5 \mathrm{~mm}$ rigid continuous flow hysteroscopic system, with a five French working channel, connected to a $12^{\circ}$ or $30^{\circ}$ optics $\left(\right.$ Olympus $^{\circledR}$, Melville, USA). Uterine distension was achieved with normal saline solution. The input pressure was kept always below $100 \mathrm{mmHg}$. Operating instruments utilized included scissors, graspers, and the bipolar Versapoint system ${ }^{\circledR}$ (Gynecare Inc.), essentially the Twizzle electrode ${ }^{\circledR}$ (Versapoint) for coagulating and cutting. Simple biopsies were performed with graspers or bipolar electrode under direct hysteroscopic guidance. Polyps and fibroids resection was achieved with scissors and mainly with bipolar device by cutting their pedicles. In broad-based and bigger polyps and fibroids, first the formation was cut into slices to easy resection and cervical canal passage. All procedures were executed by the same group of four surgeons.

Patients were admitted to the ambulatory unit and were discharged between $15 \mathrm{~min}$ and $1 \mathrm{~h}$ after surgery, depending on their recovery. The pain was assessed by a visual pain score list, graded from 0 to 10 , which was registered after each procedure.

Data were entered into an Excel 2002 ${ }^{\circledR}$ (Microsoft Inc.) database and statistical analysis was performed with SPSS 12.0 ${ }^{\circledR}$ (Statistical package for social sciences, for Windows). Continuous variables were evaluated with indepen- dent samples $t$ test and Mann-Whitney $U$ test, and for categorical variables we used the $\chi^{2}$ test, the Fisher exact test, and the Kruskal-Wallis test (ordinal variables). We considered statistical significance for a $p$ value below 0.05 .

\section{Results}

From the 245 hysteroscopic procedures evaluated, 204 $(83.3 \%)$ were classified as operative and $41(16.7 \%)$ as diagnostic. The proportion of operative hysteroscopy was identical in the two groups: $83.7 \%(144 / 172)$ in group A and $82.2 \%(60 / 73)$ in group B.

Mean age of the study patients was $65.9 \pm 9.46$ SD years, minimum 46 and maximum 89 . Table 1 presents population characteristics distribution according to study groups. Mean age was higher in women with symptomatic thickened endometrium (67.9 years old) than in asymptomatic women (64.9 years old) $(p<0.05)$. The percentage of postmenopausal women under hormonal replacement therapy (HRT) was similar in the two groups, but tamoxifen users for breast cancer chemoprophylaxis were significantly more common among asymptomatic patients $(13.4 \%$ versus $2.7 \%, p<0.05)$.

For procedure preparation, the majority $(92.3 \%)$ of the two groups' women got vaginal misoprostol pre-operatively ( $200 \mu \mathrm{g}$ on the previous day) and one third also had a nonsteroid anti-inflammatory drug (400 $\mathrm{mg}$ of ibuprofen) (Table 2). Local cervical anesthesia (5 cc of $1 \%$ lidocaine without adrenaline) was administered in $3.5 \%$ of the patients in group A and in $13.7 \%$ of those in group B ( $p>$ 0.05 , non-significant).

Hysteroscopic findings are shown in Table 3. The most frequent hysteroscopic diagnosis in both groups was apparently benign endometrial polyp. In group A, benign endometrial polyps occurred in $69.8 \%(120 / 172)$ of the patients, 108 cases $(62.8 \%)$ as isolated lesion, and 12 cases

Table 1 Population characteristics comparing asymptomatic (Group A) with symptomatic (Group B) patients

\begin{tabular}{|c|c|c|c|}
\hline & $\begin{array}{l}\text { Group A } \\
(n=172)\end{array}$ & $\begin{array}{l}\text { Group B } \\
(n=73)\end{array}$ & $\begin{array}{l}\text { Statistical } \\
\text { significance }\end{array}$ \\
\hline $\begin{array}{l}\text { Mean patient's } \\
\text { age (years) }\end{array}$ & $64.9 \pm 9.3 \mathrm{SD}$ & $67.9 \pm 9.6 \mathrm{SD}$ & $p=0.02 * *$ a \\
\hline $\begin{array}{l}\text { Patients under } \\
\text { hormonal therapy }\end{array}$ & $19.8 \%$ & $9.6 \%$ & $p>0.05^{*} \mathrm{~b}$ \\
\hline $\mathrm{HRT}^{\mathrm{c}}$ & $6.4 \%$ & $6.8 \%$ & $p>0.05^{*} \mathrm{~b}$ \\
\hline Tamoxifen therapy & $13.4 \%$ & $2.7 \%$ & $p=0.01 * * \mathrm{~b}$ \\
\hline
\end{tabular}

${ }^{*} p$ value non-significative, $* * p$ value significative

${ }^{\mathrm{a}} t$ test

${ }^{\mathrm{b}}$ Fisher exact test

${ }^{\mathrm{c}}$ Hormonal replacement therapy 
Table 2 Hysteroscopy preparation data among the two study groups: asymptomatic (group A) and symptomatic (group B) patients

\begin{tabular}{lccc}
\hline & Total $(n=245)$ & Group A $(n=172)$ & Group B $(n=73)$ \\
\hline Misoprostol & $58.8 \%$ & $58.1 \%$ & $60.3 \%$ \\
Misoprostol+NSAI & $33.5 \%$ & $37.3 \%$ & $24.7 \%$ \\
NSAI & $2.5 \%$ & $1.7 \%$ & $4.1 \%$ \\
Unknown & $3.2 \%$ & $2.9 \%$ & $4.1 \%$ \\
None & $2.0 \%$ & - & $6.8 \%$ \\
\hline
\end{tabular}

(7\%) in association with other benign findings. In group B, $65.7 \%(48 / 73)$ of the women had hysteroscopic diagnosis of endometrial polyp, being 43 cases $(58.9 \%)$ isolated and five cases $(6.8 \%)$ in association. Other benign lesions were diagnosed in $15.7 \%$ (27/172) of asymptomatic patients and in $9.6 \%(7 / 73)$ of symptomatic ones. The hysteroscopic suspicion of endometrial carcinoma occurred more frequently among symptomatic women, 17.8\% (13/73) versus $2.9 \%(5 / 172)$ of the cases, respectively, for groups B and A $(p<0.05)$.

Table 4 presents the histopathology of obtained samples. In $62.1 \%$ a benign endometrial polyp was the histological diagnosis, with no significant difference between the two study groups.

Regarding endometrial carcinoma, all the five suspected cases in asymptomatic women were confirmed, and 11 of the 13 suspected cases in symptomatic patients were histologically confirmed. The two remaining cases were endometrial hyperplasias (one simple hyperplasia and one atypical complex hyperplasia). In group $\mathrm{B}$, one case of endometrial carcinoma was histologically diagnosed, without previous hysteroscopic suspicion, which appeared on an apparently benign polyp. So, in this study, hysteroscopy showed sensitivity, specificity, positive predictive value, and negative predictive value of $94.1 \%, 98.95 \%, 88.9 \%$, and $99.5 \%$, respectively, for endometrial carcinoma diagnosis.

The histopathological diagnosis of endometrial hyperplasia was also more frequent among symptomatic patients, however without statistical significance (group A, 3.5\% versus $8.2 \%$ in group $\mathrm{B}, p>0.05$ ). The endometrial hyperplasia diagnosis was suspected on hysteroscopy but not confirmed in three cases. Two cases of hyperplasia occurred in suspected carcinomas; one case appeared after guided biopsy to apparent intrauterine adhesion area and the remaining nine cases were diagnosed in apparently benign polyps. All hyperplasias appeared among patients that were not under tamoxifen therapy.

Overall, the concordance between hysteroscopic findings and histological diagnosis was of $89.9 \%$ (187 cases in 208 applicable). See Table 5. From the apparently benign polyps on hysteroscopy, 91\% (151/166) were confirmed with pathology. There was one case of polyp with focal carcinoma $(0.6 \%)$ among asymptomatic women and nine cases of polyps with focal endometrial hyperplasia (5.4\%) six in asymptomatic patients (two with atypical hyperplasia and four with complex hyperplasia) and three in symptomatic patients (two with atypical hyperplasia and one simple hyperplasia). Hysteroscopy had sensitivity, specificity, positive predictive value, and negative predictive value of

Table 3 Endoscopic diagnosis: comparison between group A (symptomatic) and group B (asymptomatic)

\begin{tabular}{|c|c|c|c|c|}
\hline & Group A $(n=172)$ & Group B $(n=73)$ & Statistical significance & Total $(n=245)$ \\
\hline Normal uterine cavity & $16(9.3 \%)$ & $3(4.1 \%)$ & $p>0.05^{*}$ a & $7.8 \%$ \\
\hline Benign isolated polyp & $108(62.8 \%)$ & $43(58.9 \%)$ & $p>0.05^{*} \mathrm{~b}$ & $61.65 \%$ \\
\hline \multicolumn{5}{|l|}{ Other benign lesions } \\
\hline Fibroid & $12(7 \%)$ & $2(2.75 \%)$ & $p>0.05^{* \mathrm{a}}$ & $5.7 \%$ \\
\hline Intrauterine adhesion & $9(5.2 \%)$ & $3(4.1 \%)$ & $p>0.05^{*}$ a & $4.9 \%$ \\
\hline Uterine septum & $6(3.4 \%)$ & $2(2.75 \%)$ & $p>0.05^{*}$ a & $3.3 \%$ \\
\hline Endometrial carcinoma & $5(2.9 \%)$ & $13(17.8 \%)$ & $p=0.03 * * \mathrm{a}$ & $7.3 \%$ \\
\hline Endometrial hyperplasia & $2(1.2 \%)$ & $1(1.4 \%)$ & $p>0.05^{* \mathrm{a}}$ & $1.2 \%$ \\
\hline \multicolumn{5}{|l|}{ Multiple benign findings } \\
\hline Benign polyp + other & $12(7 \%)$ & $5(6.8 \%)$ & $p>0.05^{* \mathrm{a}}$ & $6.95 \%$ \\
\hline Other & $2(1.2 \%)$ & $1(1.4 \%)$ & $p>0.05^{*}$ a & $1.2 \%$ \\
\hline
\end{tabular}

${ }^{*} p$ value non-significative, ${ }^{* *} p$ value significative

${ }^{\text {a }}$ Fisher exact test

${ }^{\mathrm{b}} \chi^{2}$ test 
Table 4 Histopathological diagnosis: comparison between group A (symptomatic) and group B (asymptomatic)

\begin{tabular}{|c|c|c|c|c|}
\hline & Group A $(n=172)$ & Group B $(n=73)$ & Statistical significance & Total $(n=245)$ \\
\hline Normal endometrium & $7(4.1 \%)$ & $5(6.8 \%)$ & $p>0.05^{* a}$ & $4.9 \%$ \\
\hline Endometrial polyp & $110(64 \%)$ & $42(57.5 \%)$ & $p>0.05^{*} \mathrm{~b}$ & $62.1 \%$ \\
\hline Fibroid & $10(5.8 \%)$ & $2(2.7 \%)$ & $p>0.05^{* a}$ & $4.9 \%$ \\
\hline Other benign lesions & $3(1.7 \%)$ & $2(2.7 \%)$ & $p>0.05^{* a}$ & $2 \%$ \\
\hline Endometrial hyperplasia & $6(3.5 \%)$ & $6(8.2 \%)$ & $p>0.05^{*}$ a & $4.9 \%$ \\
\hline Endometrial carcinoma & $5(2.9 \%)$ & $12(16.4 \%)$ & $p=0.03 * *$ a & $6.9 \%$ \\
\hline No sample & $27(15.7 \%)$ & $4(5.5 \%)$ & $p=0.03 * *$ a & $12.7 \%$ \\
\hline Insufficient for diagnosis & $4(2.3 \%)$ & - & - & $1.6 \%$ \\
\hline
\end{tabular}

${ }^{*} p$ value non-significative, ${ }^{* *} p$ value significative

${ }^{\mathrm{a}}$ Fisher exact test

${ }^{\mathrm{b}} \chi 2$ test

$98.7 \%, 72.7 \%, 91 \%$, and $95.2 \%$, respectively, for benign polyp diagnosis.

No biopsy was performed in 27 cases of group A (12 normal cavity, two little polyps that were fulgurated, two fibroids, seven intrauterine adhesions, and four uterine septum) and four cases of group B (one normal cavity, two intrauterine adhesions, and one uterine septum).

After hysteroscopic diagnosis of endometrial polyp, we performed its complete excision in $91.1 \%$ of the cases $(153 /$ $168)-116 / 120$ of asymptomatic (96.7\%) and $45 / 48$ in symptomatic (93.8\%). Partial polypectomy was performed in $4.8 \%$ (eight), guided biopsy in $2.9 \%$ (five), and in $1.2 \%$ (two) sample was not obtained because we fulgurated the polyp. Versapoint ${ }^{\circledR}$ bipolar electrode was used in $92.8 \%$ of operative hysteroscopies for polyps.

Myomectomy was performed $81.8 \%(18 / 22)$ of the cases (14 isolated fibroids and eight associated). In the remaining cases, intervention was not feasible or was not necessary.

The mean procedure duration was significantly longer in group B with 36.1 min versus $31.5 \mathrm{~min}$ in group A $(p<$ $0.05)$. Regarding pain subjective evaluation, the mean score was 4 in both groups. See Table 6. There were no complications related to procedure recorded in both groups.

\section{Discussion}

Transvaginal ultrasound is a valuable identification method of women at risk for endometrial disease not only by measuring the endometrium thickness but also by accessing focal abnormalities within the endometrial cavity. Recent meta-analysis [2,9] concluded that the negative predictive value of a thin endometrium (less than $5 \mathrm{~mm}$ ) is very high among women with postmenopausal bleeding. Hydrosonohysterography allows better visualization of focal lesions within the cavity but is more painful than the sonography alone and cannot reliably discriminate between benign and malignant focal lesions [6].

The purpose of investigating postmenopausal women with thickened endometrium is essentially to exclude endometrial cancer, thus obtaining sample(s) for histopathologic examination is fundamental and obligatory. Blind endometrial biopsies (obtained with dilatation/curettage or less invasive methods such as Vabra or Pipelle) were the classical methods for obtaining endometrial samples. These blind methods are associated with low-sensitivity diagnostic rates for benign and malignant endometrial pathology [5, 6]. Nowadays, office hysteroscopy is considered the gold-
Table 5 Histological diagnosis according to previous endoscopy findings

\begin{tabular}{llllll}
\hline & \multicolumn{2}{l}{ Histology } & & \\
\cline { 2 - 5 } & Carcinoma & Hyperplasia & Benign polyp & Other benign & No pathology \\
\hline Endoscopy & & & & & \\
Carcinoma & $\mathbf{1 6}$ & 2 & - & - & 2 \\
Hyperplasia & - & - & 1 & 3 & 2 \\
Benign polyp & 1 & 9 & $\mathbf{1 5 1}$ & $\mathbf{1 3}$ & 1 \\
Other benign & - & 1 & 1 & - & $\mathbf{5}$ \\
No pathology & - & - & - & & - \\
\hline
\end{tabular}


Table 6 Hysteroscopy duration and associated pain in asymptomatic (group A) and symptomatic (group B) patients

\begin{tabular}{|c|c|c|c|}
\hline & Group A $(n=172)$ & Group B $(n=73)$ & Statistical significance \\
\hline Mean procedure duration & $31.5 \pm 14.7$ SD [CI 29.1-33.9] & $36.1 \pm 15.7$ SD [CI 32.2-40] & $p=0.028 * * \mathrm{a}$ \\
\hline Median pain (scale 1-10) & 4.0 & 4.0 & $p>0.05^{*} \mathrm{~b}$ \\
\hline
\end{tabular}

${ }^{*} p$ value non-significative, ${ }^{* *} p$ value significative

${ }^{\text {a } M a n n-W h i t n e y ~} U$ test

${ }^{\mathrm{b}}$ Kruskal-Wallis test

standard procedure for evaluating uterine cavity, allowing not only eye-guided biopsies to suspicious areas but also operative techniques for benign pathology whenever necessary [5-7].

In our study, we evaluated 245 women submitted to office hysteroscopy after sonographic diagnosis of endometrial thickening and compared asymptomatic with symptomatic patients (with postmenopausal bleeding) in respect to hysteroscopic and histopathological findings.

The symptomatic patients were significantly older and, despite identical global rates of patients under HRT, a higher number of asymptomatic women were under tamoxifen therapy (probably reflecting their rigorous breast cancer follow-up).

Pre-procedure medical preparation depended on operator option. In our institution, the protocol recommends misoprostol preparation in postmenopausal women as a cervical primer, so only a small group of patients did not administer misoprostol (less than 8\%). Cervical local anesthesia was also preformed in a minority of both groups' patients (depending on operator's option).

The most frequent hysteroscopic and histopathological diagnosis was endometrial polyp in both groups $(69.8 \%$ and $65.7 \%$ ). See and treat in one session was achieved for the majority of benign endometrial polyps and fibroids, as operative office hysteroscopy was feasible, mainly due to bipolar Versapoint ${ }^{\circledR}$ device utilization.

There is evidence that all intrauterine polypoid lesions of symptomatic postmenopausal women should be removed completely. Endometrial cancer may be confused with benign polyp during hysteroscopy, so one cannot be sure of polyp diagnosis until it is totally removed $[6,10]$. In our study, $5.4 \%$ of apparently benign polyps on hysteroscopy effectively had focal hyperplasia on pathology and $0.6 \%$ had focal carcinoma. Endometrial hyperplasia, especially atypical hyperplasia (a well-known risk factor for endometrial cancer) occurred in apparently benign polyps of both symptomatic and asymptomatic women. This aspect highlights the importance of total polyp excision after its diagnosis, even in asymptomatic patients. Additionally, endometrial polyp malignant degeneration is always a possibility to consider, occurring in $0.5 \%$ to $4.8 \%$ of the cases [6].
Loizzi et al. [10] reported a rate of $28 \%$ of hysteroscopic endometrial pathology in a group of 129 asymptomatic women with endometrium thickness superior to $4 \mathrm{~mm}$. In our study, asymptomatic patients had a higher pathology rate, $77.9 \%$, perhaps reflecting the higher mean patients' age and the better sonographic accuracy of new equipment. Also, Schmidt et al. [11], in a recently published article, found that in $79.3 \%$ of 241 patients with asymptomatic endometrial thickness (more than $6 \mathrm{~mm}$ ) an endometrial polyp was present and that in these patients the endometrial thickness was significantly higher $(12 \pm 7.4 \mathrm{~mm})$. We did not evaluate the hysteroscopic/histological data regarding the value of sonographic endometrial thickness but considering a different cut-off for asymptomatic women is a subject of debate.

Global endometrial carcinoma rate in our population (254 women with endometrial thickening) was $6.9 \%$ (17 cases) but as expected symptomatic women had a significantly higher incidence of carcinoma ( $16.4 \%$ versus $2.9 \%)$. Ribeiro et al. [12] reported only one carcinoma $(0.25 \%)$ in 399 asymptomatic patients who underwent hysteroscopy and questioned the utility of ultrasound among asymptomatic women. Our experience, on the other hand, favors sonographic screening in these women since we found higher rates of carcinoma and endometrial hyperplasia $(2.9 \%$ and $3.5 \%$, respectively). A significantly higher number of asymptomatic patients were under tamoxifen therapy in our study but all hyperplasia cases appeared in non-medicated women.

Hysteroscopy had a high specificity and negative predictive value for diagnosing carcinoma and benign polyps, with identical values described in literature [13, 14]. Only one case of carcinoma was misdiagnosed in a polyp with focal carcinoma. Regarding endometrial hyperplasia, we had worst results. The three suspected cases were not confirmed and 12 cases occurred without hysteroscopic suspicion. Several studies evaluated the accuracy of hysteroscopy in the diagnosis of endometrial hyperplasia [14-19] and all of them emphasize the lack of precise hysteroscopic criteria for hyperplasia diagnosis. In fact, our results probably underline this aspect. Pathologic exclusion of hyperplasia should be performed in all cases of thick or irregular endometrium with guided biopsy. 
Symptomatic patients had significantly longer mean procedure duration, despite identical rates of operative hysteroscopy, namely operative polypectomy. This aspect can be related to additional technical difficulties in older patients in group A or to dimension of the lesions (aspect not included in the study). On the other hand, this difference on procedure duration was not reflected on subjective pain evaluation, since the median value was identical in both groups. The majority of patients considered the procedure discomfort tolerable (median 4, in maximum of 10).

\section{Conclusions}

Endometrial thickening associated with postmenopausal metrorrhagia gives patients a higher risk for endometrial neoplasia and hyperplasia, compared with asymptomatic patients. Current hysteroscopic technology allows a rapid, safe, tolerable, and effective ambulatory technique which offers the opportunity for the diagnosis and, nearly always, treatment of endometrial benign pathology after menopause.

Conflict of interest There is no actual or potential conflict of interest in relation to this article.

\section{References}

1. Creasman WT (1997) Endometrial cancer: incidence, prognostic factors, diagnosis, and treatment. Semin Oncol 24(1)Suppl 1:S1140-S1-50

2. Smith-Bindman R, Kerlikowske K, Feldstein VA et al (1998) Endovaginal ultrasound to exclude endometrial cancer and other endometrial abnormalities. J Am Med Assoc 280:1510-1517

3. Bree RL, Bowerman RA, Bohm-Velez M et al (2000) US evaluation of the uterus in patients with postmenopausal bleeding: a positive effect on diagnostic decision making. Radiology 216:260-264
4. Bree RL, Carlos R (2002) US for postmenopausal bleeding: consensus development and patient-centered outcomes. Radiology 222:595-598

5. Bettochi S, Nappi L, Ceci O et al (2004) The role of office hysteroscopy in menopause. J Am Assoc Gynecol Laparosc 11:103-106

6. Bettocchi S, Nappi L, Ceci O et al (2005) Hysteroscopy and menopause: past and future. Curr Opin Obstet Gynecol 17 (4):366-375

7. Farrugia M, McMillan DL (2000) Versapoint in the treatment of focal intra-uterine pathology in an outpatient clinic setting. Gynecol Obstet 7:169-173

8. Vleugels MPH (2001) Normal saline field bipolar electrosurgery in hysteroscopy: report of the first 163 cases. Gynaecol Endosc 10:349-353

9. Gupta JK, Chien PF, Voit D et al (2002) Ultrasonographic endometrial thickness for diagnosing endometrial pathology in women with post-menopausal bleeding: a meta-analysis. Acta Obstet Gynecol Scand 81:799-816

10. Loizzi V, Bettocchi S, Vimercati A et al (2000) Hysteroscopic evaluation of menopausal women with endometrial thickness of $4 \mathrm{~mm}$ or more. J Am Assoc Gynecol Laparosc 7:191-195

11. Schmidt T, Breidenbach M, Nawroth F et al (2009) Hysteroscopy for asymptomatic postmenopausal women with sonographically thickened endometrium. Maturitas. doi:10.1016/j.maturitas.2008.11.018

12. Ribeiro CT, Rosa-e-Silva J, Silva-de-Sá M et al (2007) Hysteroscopy as a standard procedure for assessing endometrial lesions among postmenopausal women. Sao Paulo Med J 125(6):338-342

13. Fay TN, Khanem N, Hosking D (1999) Out-patient hysteroscopy in asymptomatic postmenopausal women. Climateric 2:263-267

14. Clark TJ, Voit D, Gupta JK et al (2002) Accuracy of hysteroscopy in the diagnosis of endometrial cancer and hyperplasia: a systematic quantitative review. JAMA 288(13):1610-1621

15. Arslan S, Aytan H, Gunyeli I et al (2005) Office hysteroscopic evaluation of endometrium: can we hit the target? Arch Gynecol Obstet 271:200-202

16. Garuti G, Cellani F, Garzia D et al (2005) Accuracy of hysteroscopic diagnosis of endometrial hyperplasia: a retrospective study of 323 patients. J Minim Invasive Gynecol 12(3):247-253

17. Vasile C, Piazza M (2003) Accuracy of office hysteroscopy in the diagnosis of endometrial hyperplasia. Clin Exp Obstet Gynecol 30 (4):223-225

18. Garuti G, Sambruni I, Colonneli M, Luerti M (2001) Accuracy of hysteroscopy in predicting histopathology of endometrium in 1500 women. J Am Assoc Gynecol Laparosc 8(2):207-213

19. De Wit A, Vleugels MPH, Kruif JH (2003) Diagnostic hysteroscopy: a valuable diagnostic tool in the diagnosis of structural intra-cavital pathology and endometrial hyperplasia or carcinoma? Six years of experience with non-clinical diagnostic hysteroscopy. Eur J Obstet Gynecol Reprod Biol 110:79-82 